\title{
PROTECTIVE EFFECT OF RESVERATROL IN COMBINATION WITH URSOLIC ACID AGAINST DIABETIC CARDIOMYOPATHY VIA IMPROVING MYOCARDIAL FUNCTION AND ATTENUATION OF INFLAMMATION AND OXIDATIVE STRESS
}

\author{
XIN SONG ${ }^{1 \#}$, GUOPENG HUANG ${ }^{2 \#}$, WEI HAN ${ }^{3}$, YONG ZHAO ${ }^{4}$ and PENG DONG ${ }^{*}$
}

${ }^{1}$ Lizhou Maternal and Child Health Hospital, Lizhou Second People \& Hospital Cardiovascular Medicine, Sichuan Province 646200, China

${ }^{2}$ Department of Cardiology, Chongqing Dazu District People's Hospital, Chongqing 402360, China

${ }^{3}$ Second Department of Cardiovasology, Foshan Chancheng Central Hospital, Foshan City, Guangdong Province 528000, China

${ }^{4}$ Department of Cardiology, The Second Affiliated Hospital of Hainan Medical College, Haikou City, Hainan Province 570203, China

${ }^{5}$ Second Department of Cardiology, Aviation General Hospital of China Medical University, Beijing 100012, China

\begin{abstract}
The present study intended to investigate the effect of resveratrol (RES) and ursolic acid (URA) against diabetic cardiomyopathy (DCM). The streptozotocin (STZ) was injected in Sprague-Dawley (SD) rats to induce DCM and then rats were treated with RES and URA at a dose of $15 \mathrm{mg} / \mathrm{kg}$ alone and in combination for 8 weeks. Afterward, the rats were examined for changes in fasting glucose, HW/BW ratio, TG, TC, ALT, AST, BUN, Cr along with biomarkers depicting oxidative stress status and inflammation. The effect was also examined on cardiac function via echocardiography and NF- $\kappa B$ and TLR4 via western blot analysis. It was found that biochemical parameters together with cardiac functions were restored near to normal more significantly in RES+URA combination than alone treated group. The level of inflammation and oxidative stress was also found to be decreased in the combination treatment group than alone. Moreover, the level of NF- $\kappa \mathrm{B}$ and TLR4 was decreased significantly in RES + URA treated group. Our results collectively suggest that resveratrol and ursolic acid in combination can be valuable in protecting DCM.
\end{abstract}

Keywords: Cardiomyopathy, cardiac function, inflammation, oxidative stress, NF- $\kappa \mathrm{B}$

Diabetes mellitus (DM) is a serious complication of persistent hyperglycemia where affected individuals experience insufficient production of insulin or inadequate utilization of glucose (1). It leads to long term disorders mainly affecting vital organs, heart (diabetic cardiomyopathy), retina (diabetic retinopathy) and kidney (nephropathy). Among these complications, the heart is more vulnerable than other organs and studies estimated that nearly $65-70 \%$ of diabetic people prone to die due to diabetic cardiomyopathy (DCM) (2-4). It is characterized by alteration in the myocardium which induces cardiomyocyte hypertrophy, myocardial fibrosis and apoptosis, where they are not directly linked to coronary artery diseases or hypertension. The etiology of DCM is multifactorial involving hyperglycemia-induced oxidative stress, inflammation, and activation of apoptosis. These processes can sooner or later lead to heart failure (5-8). Various agents are used in clinical practice to counteract the menace of DCM, however, no single agent was proved effective till now.

Natural products have proved their significance in modern medicine and as a result, nearly $35 \%$ of the global medicine market has been occupied by products derived from them $(9,10)$.

* Corresponding author: e-mail: dongpeng361@sina.com 
Resveratrol (RES), a naturally occurring stilbene, is found in a variety of plant species, such as grapes and groundnuts, and exhibit various pharmacological properties. Interestingly, diverse reports have documented the protective effect of RES against DCM via multiple mechanisms, such as modulation of Sirtuin 1 (Sirt1), Sirt3, tumor necrosis factor- $\alpha$ $(\mathrm{TNF}-\alpha)$ /nuclear factor-kappa B (NF- $\kappa \mathrm{B})$ signaling and NF-E2-related factor 2 (Nrf2) (11-17). Moreover, Ursolic acid (URA), a pentacyclic triterpenoid compound, naturally occurring in food products such as apples and basil recently showed promising activity against DCM in the murine model (1820). Thus, prompted by the above and excellent cardioprotective activity of RES and URA, the present study was intended to scrutinize the pharmacological benefit of RES + URA after combination in the murine model of DCM.

\section{EXPERIMENTAL}

Animal preparations and experimental procedure

Six weeks old male Sprague-Dawley rats, weighing 160-170 g, were obtained from the institutional animal house. Rats were housed in a laboratory environment in strict hygienic conditions. Initially, 10 rats in a random manner were fed a normal diet, where other rats were allowed to eat a high-sucrose-fat diet during the entire experiment period for 8 weeks before injection. The rats were starved overnight before the induction of diabetes by intravenous administration of STZ $(30 \mathrm{mg} / \mathrm{kg}$, Sigma). The rats whose blood glucose level was found to be $=16.7 \mathrm{mmol} / \mathrm{L}$ at in $3^{\text {rd }}$ and $7^{\text {th }}$ day after STZ injection were selected for the study. After that, the rats were randomly categorized into five treatment groups, as follows, sham as normal saline, disease control with DM, RES (15 mg/kg), URA (15 $\mathrm{mg} / \mathrm{kg}$ ) and RES + URA (15 mg/kg each). The level of blood glucose was recorded. Moreover, at the end of the study, echocardiography was conducted to assess cardiac function, and then rats were euthanized. The blood and heart were collected for biochemical analysis. The ratio of heart weight/body weight $(\mathrm{HW} / \mathrm{BW})$ was performed at the time of sacrificing. The study protocols were approved by the Animal Care and Use Committee of the Institute. All procedures and protocols followed the guidelines in the National Institutes of Health Guide for the Care and Use of Laboratory Animals.

\section{Hemodynamic index}

The hemodynamic index, such as left ventricular systolic pressure (LVSP), left ventricular end- diastolic pressure (LVEDP) and the increase rate of the left ventricular pressure $\left( \pm d p / d t_{\max }\right)$ of rats was recorded on a four-channel acquisition system (BL420S; Chengdu Taimeng Software Co. Ltd., Chengdu, China).

\section{Biochemical characteristics in serum and heart tissue}

The serum and myocardial tissues homogenate obtained from rats were used for the determination of various bio-markers as per the manufacturer's protocol. The level of fasting insulin was recorded by ELISA (Jiangsu Enzyme Free Experimental Co., Ltd. China). The level of total cholesterol (TC), total triglycerides (TG), blood urea nitrogen (BUN), aspartate aminotransferase (AST), alanine aminotransferase (ALT), and creatinine (Cr) were determined using commercial assay kits (Nanjing Jiancheng BioTech. Co. Ltd, Jiangsu, China)

\section{Evaluation of MDA, GSH and SOD}

The level of MDA, GSH and SOD were determined by commercially available ELISA (Jiangsu Enzyme Free Experimental Co., Ltd. China) kits as per the manufacturer protocol.

\section{Evaluation of pro-inflammatory cytokines}

The level of TNF- $\alpha$, IL- $1 \beta$ and IL-6 were determined by commercially available ELISA kits as per the manufacturer protocol (Jiangsu Enzyme Free Experimental Co., Ltd. China).

\section{Western blotting}

The protein extract $(30 \mu \mathrm{g})$ was resolved by $12 \%$ SDS-PAGE electrophoresis and electrotransferred onto a membrane of nitrocellulose. The membrane was blocked-up using TrisPBS (TPBS) with Tween 20 and 5\% skimmed milk and probed with primary antibodies: Next, the secondary antibody conjugated to HRP (1: 10 000; Abcam/Cell Signaling Technology) in TPBS was added and incubated at $37^{\circ} \mathrm{C}$ for $1 \mathrm{~h}$. Bands were visualized using an enhanced chemiluminescence image analyzer, and protein levels were quantified using Image-Pro Plus software (Media Cybernetics, Inc., MD, USA).

\section{Statistical analysis}

The data were articulated as mean \pm standard error (SEM). The statistical analysis was performed using one-way analysis of variance (ANOVA) followed by Bonferroni post hoc multiple comparison test (GraphPad Prism 5.0, USA). The P-value $<0.05$ was considered as statistically significant. 
A

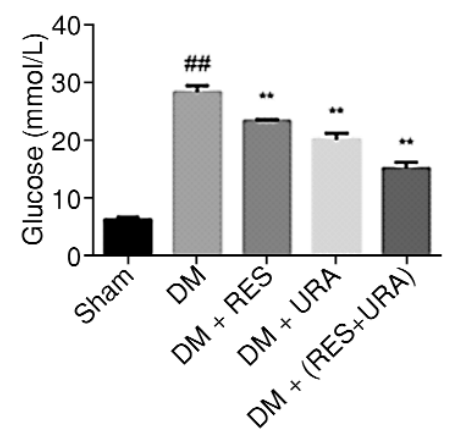

C

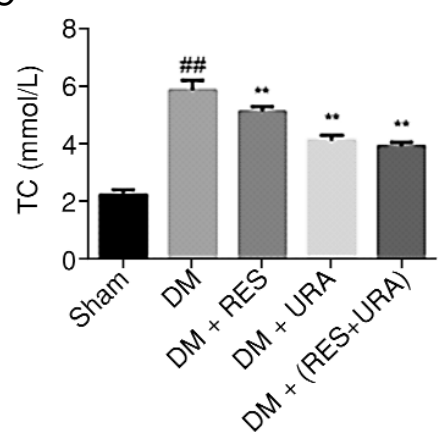

E

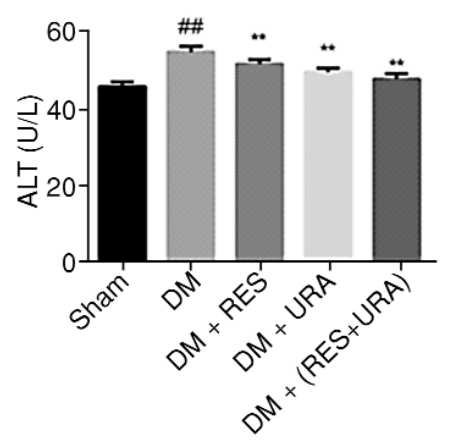

G

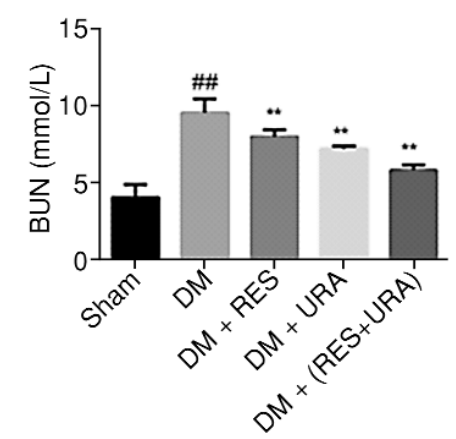

B

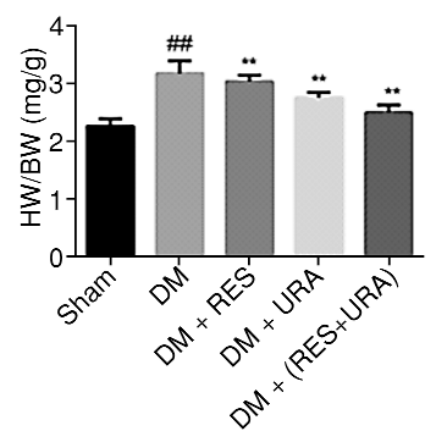

D

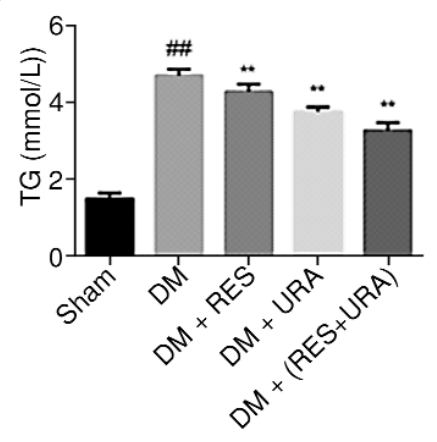

F

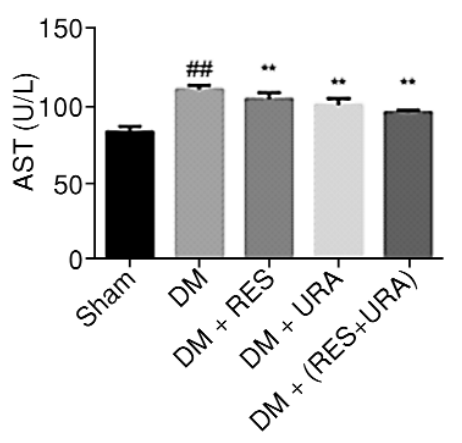

$\mathrm{H}$

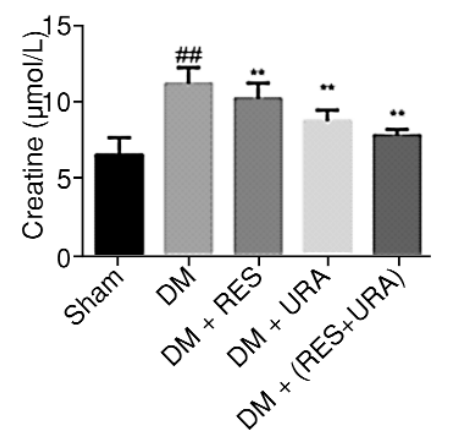

Figure 1. Effect of RES (15 mg/kg) and URA $(15 \mathrm{mg} / \mathrm{kg})$ alone and in combination on various biological parameters in the STZ-induced $\mathrm{DCM}$ rats. Values represent the mean \pm SEM and are representative of three independent experiments. \#\#\# $<0.05 \mathrm{vs}$ control; **P $<0.05$, one-way analysis of variance (ANOVA) followed by Bonferroni post hoc test. 


\section{RESULTS}

\section{Effect on the basic biological parameter}

As presented in Figure 1, the effect of RES and URA alone and in combination was determined on the basic biological parameters of rats after induction of DCM using STZ. The plasma glucose level together with HW/BW ratio was found elevated in the DM group as compared to the sham. Moreover, the lipid level (TC and TG) along with serum kidney (BUN and Cr) and liver (AST and ALT) function were found to be elevated significantly in the DM group as compared to Sham. On closely inspecting Figure 1, it could be summarized that RES + URA showed more pronounced restoration of these biomarkers near to normal than alone treated group.

\section{Effect on the cardiac function}

The effect of test drugs was assessed on the cardiac function of DCM rats by studying left ventricular hemodynamic parameters. As shown in
Figure 2, the LVSP, $+\mathrm{dp} / \mathrm{dt}_{\max }$ and $-\mathrm{dp} / \mathrm{dt}_{\max }$ of the disease group were drastically declined, while the LVEDP values were appreciably augmented as compared to control. These significant alterations in the hemodynamic parameters suggested cardiac insufficiency in the DCM rats. Moreover, upon treatment with RES or URA, these parameters were significantly improved. The more prominent activity was achieved in the case of RES along with the URA treated group.

\section{Effect on oxidative stress}

In the next phase, the effect was quantified on the oxidative stress biomarkers. As presented in Figure 3, the disease group showed a reduction in SOD and GSH level together with an increase in MDA activity. The level of these biomarkers was restored near to normal in the treated group with RES or URA as compared to the disease group. Moreover, in a combination of RES with URA, a significant reduction in oxidative stress was observed.
A

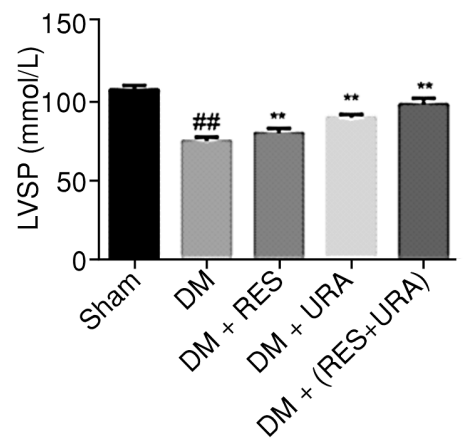

C

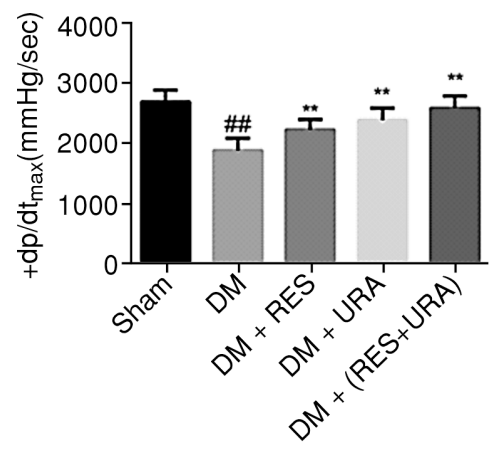

B

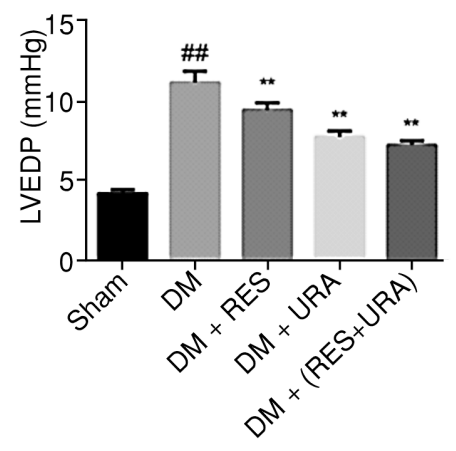

D

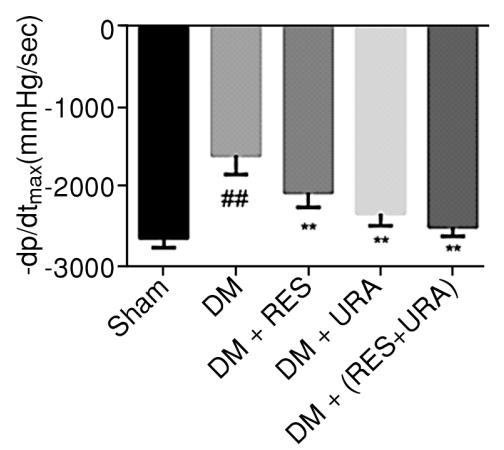

Figure 2. Effect of RES (15 mg/kg) and URA (15 mg/kg) alone and in combination on the cardiac function in DCM rats. (a) LVSP (b) LVEDP (c) $+d p / d t m a x$ and (d) - dp/dtmin. Values represent the mean \pm SEM and are representative of three independent experiments. \#\#\#P $<0.05 \mathrm{vs}$ control; **P $<0.05$, one-way analysis of variance (ANOVA) followed by Bonferroni post hoc test. 
A

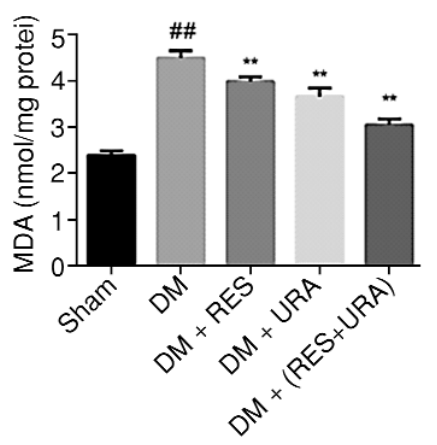

B

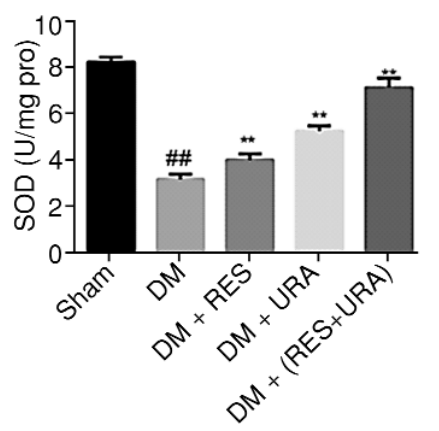

C

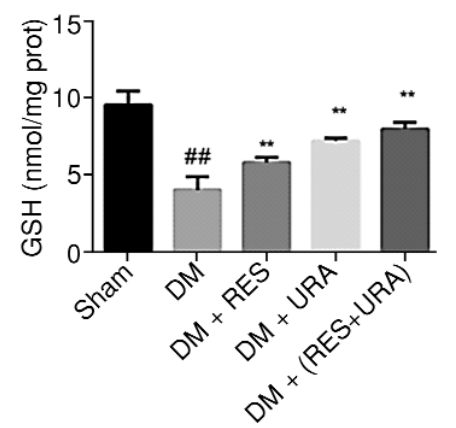

Figure 3. Effect of RES (15 mg/kg) and URA (15 mg/kg) alone and in combination on the oxidative stress biomarkers. (a) MDA (b) SOD and (c) GSH in DCM rats. Values represent the mean \pm SEM and are representative of three independent experiments. \#\#\#P $<0.05$ vs control; **P $<0.05$, one-way analysis of variance (ANOVA) followed by Bonferroni post hoc test.

A

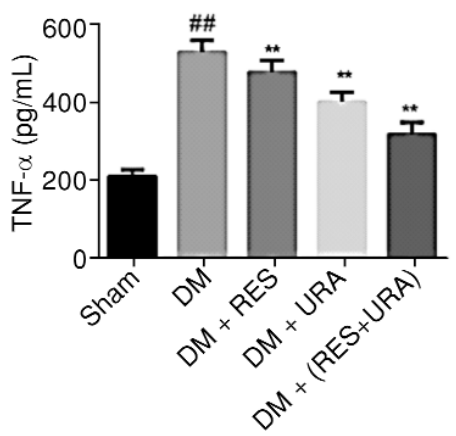

B

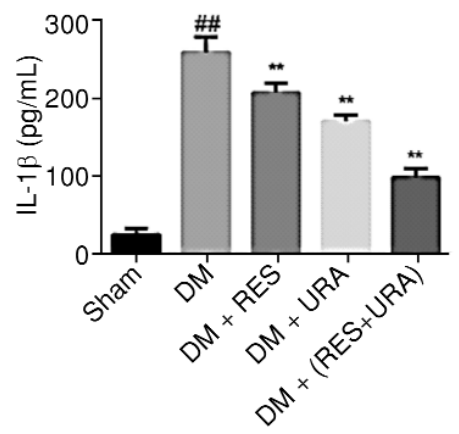

C

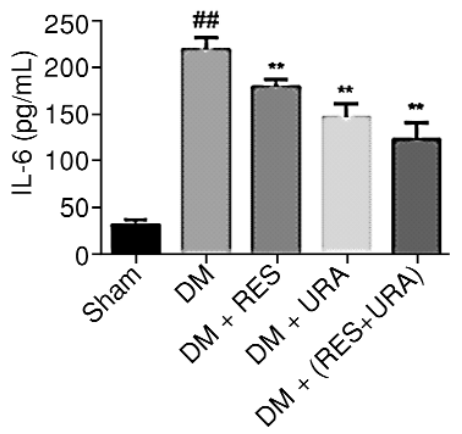

Figure 4. Effect of RES (15 mg/kg) and URA ( $15 \mathrm{mg} / \mathrm{kg}$ ) alone and in combination on the pro-inflammatory cytokines (a) TNF- $\alpha$ (b) IL1 and (c) IL-6. Values represent the mean \pm SEM and are representative of three independent experiments. \#\#\#P $<0.05$ vs control; $* * P<$ 0.05 , one-way analysis of variance (ANOVA) followed by Bonferroni post hoc test.

\section{Effect on pro-inflammatory cytokines}

As presented in Figure 4, as compared to sham, the concentration of various pro-inflammatory cytokines TNF- $\alpha$, IL- $1 \beta$ and IL- 6 was found elevated in DM group. Moreover, in RES or URA treated group, the level of these cytokines was reduced noticeably. A much significant reduction in the level of these cytokines was observed in RES + URA group.

\section{Effect on the NF- $\mathrm{KB}$ pathway}

The effect of compounds was assessed on the $\mathrm{NF}-\kappa \mathrm{B}$ and TLR4 signaling mediators of inflammation via western blot. As shown in Figure 5, as compared to sham, the level of these mediators was found elevated in DM rats. In the group treated with
RES and URA, a considerable decrease in the expression of both NF- $\kappa \mathrm{B}$ and TLR4 was observed, which was further reduced in RES + URA group.

\section{DISCUSSION}

Diabetic cardiomyopathy (DCM) is a serious complication among the person affected by diabetes and is considered to be responsible for the majority of heart-related disorders (7). The RES and URA were proved as effective agents to mitigate the consequence of DCM via multiple mechanisms. It has been shown that RES serves as a modulator of diverse molecular signaling pathways, such as MAPK, Akt, AMPK, and Sirt1/3 pathways against DCM. The RES also showed to attenuate oxidative 
stress, inflammation, apoptosis, and autophagy, which inhibit cardiac hypertrophy and ultimately progress cardiac function in diabetes (21). On the other hand, in various studies, URA prevented STZinduced diabetic cardiomyopathy by anti-inflammatory, anti-oxidant and anti-fibrosis responses. Moreover, URA showed to inhibit myocardial fibrosis and cardiac inflammation, which results in better left ventricular function in the diabetic rats via attenuation of oxidative stress, MCP- 1 , TGF- $\beta 1$, TNF- $\alpha$ and up-regulating the expressions of MMP-2 in the myocardium $(19,20,22)$. In response to this, the present study was the first to report the effect of RES and URA alone and in combination. Our study demonstrated that effect is more pronounced against DCM when both drugs are combined than alone. They are suggested to improve biochemical parameters and cardiac function preventing left ventricular failure. They are also suggested to mitigate the oxidative stress and inflammation via inhibition of $\mathrm{NF}-\kappa \mathrm{B}$. Taken together, our findings suggested that the combination of RES with URA treatment might provide a promising strategy for attenuation of DCM.

It has been reported that patients with DM have serious complications affecting heart, kidney and liver due to severe hyperglycemic state (23).
Therefore, the therapeutic modalities to treat DM are concentrated towards limiting hyperglycemia and also to provide protection to these vital organs. In the present study, the combination of RES and URA showed to reduce blood glucose level and improved renal and hepatic function. The effect of RES and URA combination is also more pronounced on the cardiac function as shown by the improvement in LVSP, LVEDP and $\pm \mathrm{dp} / \mathrm{dt}$. Various studies have shown an intriguing correlation between oxidative stress and inflammation in DCM (24-27). Studies showed that the generation of oxidative stress promotes the secretion of various pro-inflammatory cytokines which causes the initiation of inflammation (28). Therefore, in the present study, it has been found that a combination of RES and URA inhibited oxidative stress along with a reduction in the level of various pro-inflammatory cytokines. The nuclear transcription factor NF- $\mathrm{BB}$, a transcription factor upon activation by external stimuli showed to regulate various genes affecting apoptosis and inflammation. Recent studies have shown that TLR4 induces NF- $\kappa \mathrm{B}$ and up-regulate various cytokines such as IL-6 and TNF- $\alpha$ in DM (29-32). Our results demonstrated that the combination of RES and URA prevented the activation of NF- $\kappa B$ and TLR4.

A

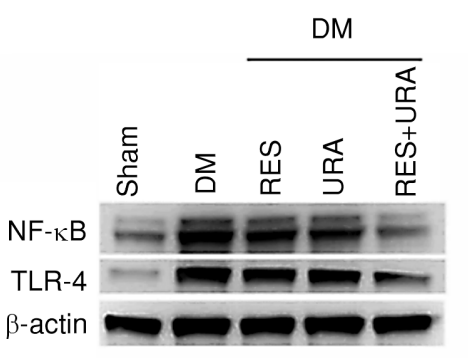

B

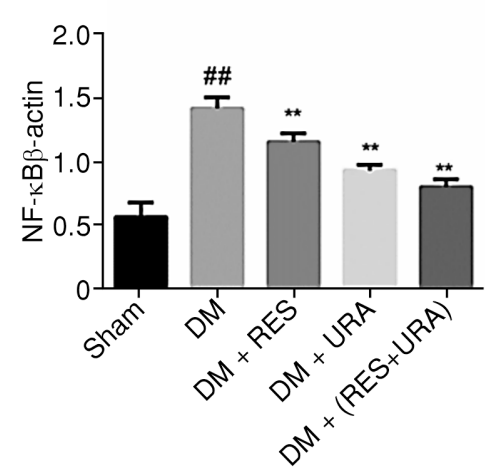

C

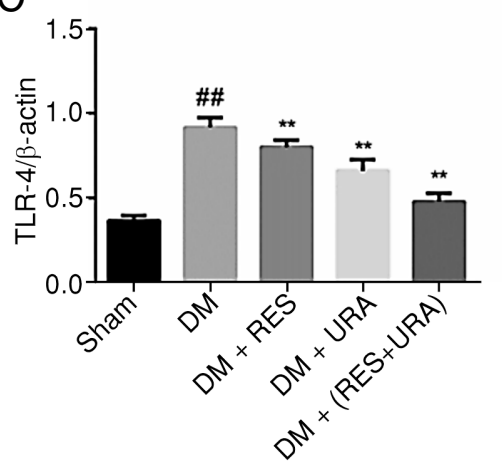

Figure 5. Effect of RES (15 mg/kg) and URA $(15 \mathrm{mg} / \mathrm{kg})$ alone and in combination on NF- $\kappa \mathrm{B}$ and TLR4 via western blot and representative bar-graph (b) NF- $\kappa B$ and (c) TLR4 in DCM rats. Values represent the mean \pm SEM and are representative of three independent experiments. \#\#\#P $<0.05$ vs control; **P $<0.05$, one-way analysis of variance (ANOVA) followed by Bonferroni post hoc test. 


\section{CONCLUSION}

Our results collectively suggest that resveratrol and ursolic acid in combination significantly inhibited myocardial inflammation and oxidative stress in diabetic rats, than alone. Moreover, it could be suggested that both RES and URA significantly attenuated TLR4 induced inhibition of NF- $\kappa B$ in DCM. The results of the present study suggest the utilization of resveratrol and ursolic acid as a promising potential therapeutics against DCM. However, further studies are warranted to define the influence of pharmacokinetics on the pharmacological effect of both drugs on each other.

\section{Acknowledgment}

The authors are thankful to Luzhou Municipal Health Bureau, Grant number: 2014245 for funding this project.

\section{Conflict of interest}

The authors declare no conflict of interest.

\section{REFERENCES}

1. Kharroubi A.T., Darwish H.M.: World J. Diabetes 6, 850 (2015).

2. Boudina S., Abel E.D.: Circulation 115, 3213 (2007).

3. Bugger H., Abel E.D.: Diabetologia 57, 660 (2014).

4. Miki T., Yuda S., Kouzu H., Miura T.: Heart Fail. Rev. 18, 149 (2013).

5. Asghar O., Al-Sunni A., Khavandi K., Khavandi A., Withers S. et al.: Clin. Sci. 116, 741 (2009).

6. Poornima I.G., Parikh P., Shannon R.P.: Circ. Res. 98, 596 (2006.)

7. Zhi Y.F., Prins J.B., Marwick T.H.: Endocr. Rev. 25, 543 (2004).

8. Huynh K., Bernardo B.C., McMullen J.R., Ritchie R.H.: Pharmacol. Ther. 142, 375 (2014)

9. Katz L., Baltz R.H.: J. Ind. Microbiol. Biotechnol. 142, 375 (2014).

10. Butler M.S., Robertson A.A.B., Cooper M.A.: Nat. Prod. Rep. 31, 1612 (2014).
11. Sulaiman M., Matta M.J., Sunderesan N.R., Gupta M.P., Periasamy M., et al.: Am. J. Physiol. Heart Circ. Physiol. 298, H833 (2010).

12. Wu H., Li G.N., Xie J., Li R., Chen Q.H., et al.: BMC Cardiovasc. Disord. 16, 5 (2016).

13. Mohammadshahi M., Haidari F., Soufi F.G.: Cardiol. J. 21, 39 (2014).

14. Fang W.J., Wang C.J., He Y., Zhou Y.L., Peng X.D., Liu S.K.: Acta Pharmacol. Sin. 39, 59 (2018).

15. Delucchi F., Berni R., Frati C., Cavalli S., Graiani G., et al.: PLoS One 7, e39836 (2012).

16. Omer Z.K., Wang Y., Liu J., Liu Y., Liu B.: African J. Pharm. Pharmacol. 5, 2483 (2011).

17. Bagul P., Katare P., Bugga P., Dinda A., Banerjee S.K.: Cells 7, 235 (2018).

18. Jang S.M., Yee S.T., Choi J., Choi M.S., Do G.M., et al: Int. Immunopharmacol. 9 113, (2009).

19. Wang X.-T., Gong Y., Zhou B., Yang J.-J., Cheng Y., et al.: Biomed. Pharmacother. 97, 1461 (2018).

20. Jang S.M., Kim M.J., Choi M.S., Kwon E.Y., Lee M.K.: Metabolism 59, 512 (2010).

21. Song Y.-J., Zhong C.-B.,Wu W.: Oxid. Med. Cell. Longev. 1, 7051845 (2020).

22. Ullevig S.L., Zhao Q., Zamora D., Asmis R.: Atherosclerosis 219, 409 (2011).

23. American Diabetes Association. Diagnosis and classification of diabetes mellitus, Diabetes Care 37, S81 (2014).

24. Giacco F., Brownlee M.: Circ. Res. 107, 1058 (2010)

25. Kayama Y., Raaz U., Jagger A., Adam M., Schellinger I.N., et al.: Int. J. Mol. Sci. 16, 25234 (2015).

26. Jia G., Hill M.A., Sowers J.R.: Circ. Res. 122, 624 (2018).

27. Faria A., Persaud S.J.: Pharmacol. Ther. 172, 50 (2017).

28. Golbidi S., Badran M., Laher I.: Exp. Diabetes Res. 2012, 941868 (2012).

29. Indira M., Abhilash P.A.: For. Immunopathol. Dis. Therap. 4, 111 (2013).

30. Zhang N., Yang Z., Xiang S.Z., Jin Y.G., Wei W.Y., et al.: Mol. Cell. Biochem. 417, 87 (2016).

31. Palomer X., Salvadó L., Barroso E., VázquezCarrera M.: Int. J. Cardiol. 168, P3160 (2013).

32. Sun D., Shen M., Li J., Li W., Zhang Y., et al.: Cardiovasc. Diabetol. 10, 4 (2011). 